\title{
A novel mutation of the $M I T F$ gene in a family with Waardenburg syndrome type 2: A case report
}

\author{
YUNFANG SHI, XIAOZHOU LI, DUAN JU, YAN LI, XIULING ZHANG and YING ZHANG
}

Medical Genetic Laboratory, Department of Obstetrics and Gynecology, Tianjin Medical University General Hospital, Tianjin 300052, P.R. China

Received October 4, 2014; Accepted January 11, 2016

DOI: $10.3892 /$ etm.2016.3042

\begin{abstract}
Waardenburg syndrome (WS) is an autosomal dominant disorder with varying degrees of sensorineural hearing loss, and accumulation of pigmentation in hair, skin and iris. There are four types of WS (WS1-4) with differing characteristics. Mutations in six genes [paired box gene 3 (PAX3), microphthalmia-associated transcription factor $(M I T F)$, endothelin 3 (END3), endothelin receptor type B (EDNRB), SRY (sex determining region Y)-box 10 (SOX10) and snail homolog 2 (SNAI2)] have been identified to be associated with the various types. This case report describes the investigation of genetic mutations in three patients with WS2 from a single family. Genomic DNA was extracted, and the six WS-related genes were sequenced using next-generation sequencing technology. In addition to mutations in PAX3, EDNRB and SOX10, a novel heterozygous MITF mutation, p. $\Delta 315$ Arg (c.944_946delGAA) on exon 8 was identified. This is predicted to be a candidate disease-causing mutation that may affect the structure and function of the enzyme.
\end{abstract}

\section{Introduction}

Waardenburg syndrome (WS) is a rare genetic disorder characterized by varying degrees of sensorineural hearing loss and accumulated pigmentation in the hair, skin and iris (1). Typically, WS is described as an autosomal dominantly inherited disorder of neural crest cells, with an incidence of 1 in 40,000 (2). The symptoms of WS are heterogeneous, and WS is classified into four major types (WS1-4), with 10 subtypes (3-6). Currently, there are six genes [paired box gene $3(P A X 3)$, microphthalmia-associated transcription factor $(M I T F)$, endothelin 3 (END3), endothelin receptor type $\mathrm{B}$

Correspondence to: Professor Ying Zhang, Medical Genetic Laboratory, Department of Obstetrics and Gynecology, Tianjin Medical University General Hospital, 154 Anshan Road, Heping, Tianjin 300052, P.R. China

E-mail: yingzhang114@163.com

Key words: MITF gene, mutation, Waardenburg syndrome, next-generation sequencing
(EDNRB), SRY (sex determining region Y)-box 10 (SOX10) and snail homolog 2 (SNAI2)] that have been reported to be involved in the pathogenesis of WS. Among these, mutations in the MITF, SOX10 and SNAI2 genes have been frequently detected in patients with WS2 (2,7-10). To date, a total of 56 mutations of the MITF gene have been summarized in the Leiden Open Variation Database (http:/grenada.lumc. nl/LOVD2/WS/), with the majority of the mutations associated with exons 7 and 8.

The present report describes three patients with WS2 from the same family, for whom next-generation sequencing technology was used to investigate the six WS-related genes in order to identify potentially pathogenic mutations.

\section{Case report}

Patients and data collection. The proband was a 29-year-old woman, who presented to the prenatal diagnosis center of the Tianjin Medical University General Hospital (Tianjin, China). The proband had sensorineural hearing loss, pigmentation on the face, blue-colored irises and premature graying of the hair. Routine audiological and ophthalmological examinations were performed. The father, mother and younger brother of the proband and 10 healthy volunteers were subsequently included in the study. Written informed consent was obtained from each participant. The protocols were approved by the Ethics Committee of the Tianjin Medical University General Hospital.

Targeted next-generation sequencing. Peripheral blood samples (5 ml) were collected from all participants. Genomic DNA was extracted from the peripheral blood using a QIAamp DNA Blood Mini kit (Qiagen GmbH, Hilden, Germany), according to the manufacturer's protocol, and then fragmented using an ultrasonoscope (Covaris S2; Covaris, Woburn, MA, USA) $(11,12)$. Examination of the genomic DNA was conducted using standard methods, including capture array design, library construction and next-generation sequencing, which followed previously described protocols $(11,12)$. In brief, 40 targeted exon sequences of the PAX3, MITF, EDN3, EDNRB, SOX10 and $S N A I 2$ genes were obtained and enriched using an array-based hybridization chip (Roche NimbleGen, Madison, WI, USA) followed by sequencing using a HiSeq2000 sequencing system (Illumina, Inc., San Diego, CA, USA) to generate paired-end reads, adhering to the standard operation protocols $(11,12)$. 
I

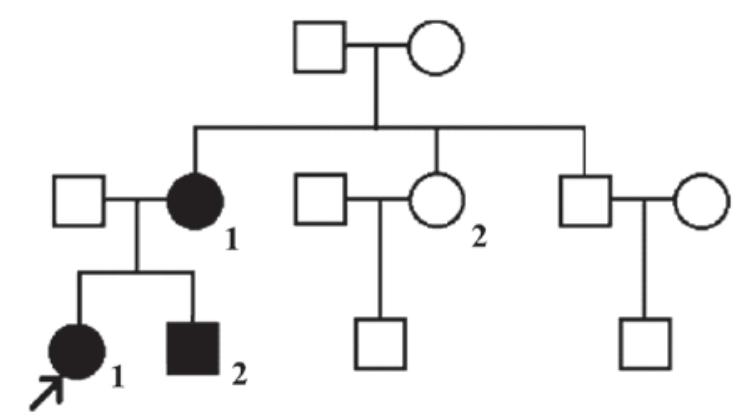

Figure 1. Pedigree chart of a family with Waardenburg syndrome type 2. The black and unfilled shapes represent affected and unaffected family members, respectively; squares represent males and circles represent females. The black arrow indicates the proband (III:1).

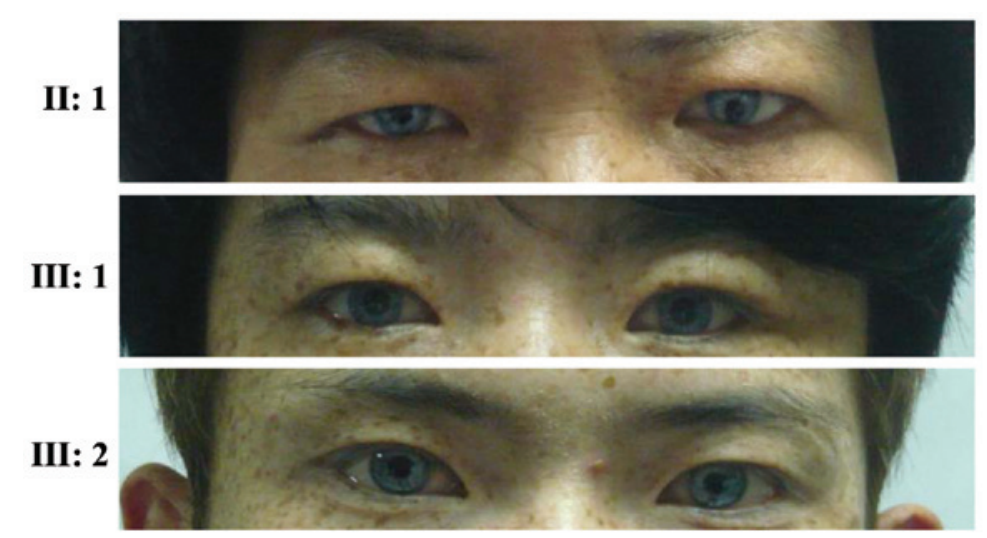

Figure 2. Partial facial photographs of three individuals affected by Waardenburg syndrome 2 showing blue irises. II:1, mother of proband; III:1, proband; III:2, younger brother of proband.

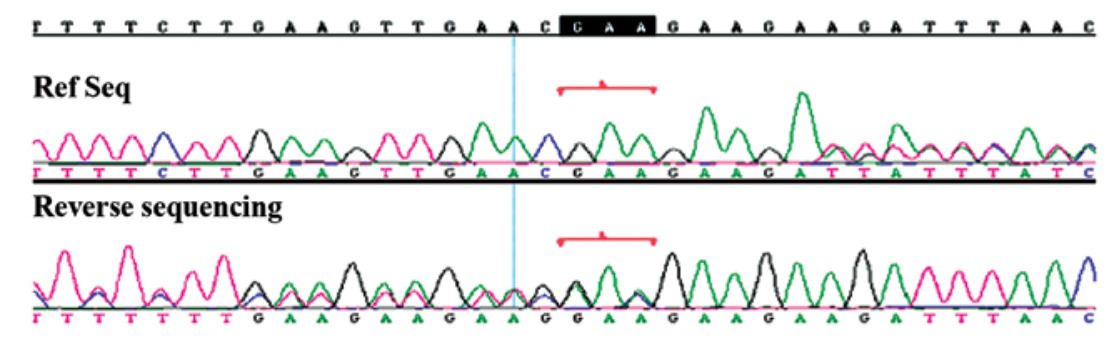

Figure 3. Sequencing of exon 8 of the MITF gene. Red brackets indicate the position of the mutation.

Short reads mapping and alignment were performed using Burrows-Wheeler Aligner software (http://bio-bwa.sourceforge. net/) (13). All primer sequences were designed on the basis of gene sequences in the NCBI database, and were synthesized by Shanghai Shangon. Co., Ltd. (Shanghai, China).

Sanger sequencing. The potentially mutated base was amplified using polymerase chain reaction $(11,12)$, and sequenced by Sanger sequencing using an ABI 3730 DNA Analyzer (Applied Biosystems; Thermo Fisher Scientific, Inc., Waltham, MA, USA). Subsequently, the similarities between the obtained sequences and the reference sequence of MITF (GenBank accession No. NM_198159) were evaluated using the Nucleotide-Nucleotide BLAST (blastn) tool.

Clinical features and family history. The proband (III:1) had sensorineural hearing loss, facial pigmentation, blue-colored irises and premature graying of the hair. However, no dystopia canthorum was observed. The detailed family history of the proband indicated that her mother (II:1) and younger brother (III:2) exhibited similar symptoms; thus, two generations of the family were affected by this condition (Figs. 1 and 2). The freckles of II:1 had lightened as the patient aged. Routine clinical examination, and audiological and ophthalmological evaluations were performed on these three individuals, and they were diagnosed with WS2 on the basis of relevant WS Consortium criteria $(2,14)$.

Mutation analysis. Mutation analysis was performed for all six pathogenic genes, which revealed no large deletions or duplications. However, a heterozygous MITF mutation p.A315Arg (c.944_946delGAA) on exon 8 was identified in all three patients (Fig. 3). A synonymous mutation (p.Gly43Gly) was identified in $P A X 3$. Two synonymous mutations, including 
p.Leu277Leu and p.Ser184Ser, were identified in the gene $E D N R B$. A synonymous mutation (p.His309His) was detected in SOX10. No synonymous gene mutations were identified in other family members and healthy volunteers.

\section{Discussion}

The symptoms of WS vary according to the type of disease. For example, sensorineural hearing loss is observed in $60 \%$ of WS1 patients and $90 \%$ of WS2 patients (7). In addition, a white forelock is presented in at least one-third of patients with WS1 or WS2 (7). In the present study, identical symptoms including sensorineural hearing loss, facial pigmentation, blue-colored irises and premature graying of hair were observed in the proband, her mother and younger brother. In the case of the mother, the symptomatic freckles were attenuated with aging.

In the majority of cases of WS, one of the proband's parents and one or more siblings are affected $(5,6)$. By analysis of the pedigree chart, it was deduced that the proband's mother had a de novo mutation and the proband and her younger brother inherited the mutation from their mother. The offspring of individuals with WS have a $50 \%$ chance of inheriting the pathogenic mutation; therefore, an exact description of the mutations responsible for the WS is crucial for the genetic counseling of WS patients and their families (4).

Although numerous patients have been diagnosed with WS2 on the basis of their clinical symptoms, the pathogenic mutations in these patients remain unclear, primarily due to the extensive diagnostic criteria for WS2 (7). In the present study, the heterozygous mutation p.A315Arg (c.944_946delGAA) was identified in the MITF gene as a genetic cause of WS2. MITF, a basic helix-loop-helix leucine zipper (bHLHZip) protein, is a transcription factor involved in numerous developmental processes; in particular, it is essential in regulating melanocyte development and the biosynthetic melanin pathway (7). In humans, MITF mutations have been reported to result in abnormal melanocyte development and WS2 (6). This particular mutation is novel in WS2 patients and could lead to a change in amino acid sequence.

In conclusion, the present study identified the heterozygous MITF mutation p.A315Arg (c.944_946delGAA) on exon 8 of three patients with WS2 from the same family, using targeted sequence capture and next-generation sequencing. The mutation may result in a truncated protein, and may affect the bHLH-ZIP structural domain. These novel results from the current study expand the known spectrum of MITF mutations.

\section{Acknowledgements}

This study was supported by a grant from the Tianjin Science and Technology Support Program (no. 11ZCGYSY02500).

\section{References}

1. Yang T, Li X, Huang Q, Li L, Chai Y, Sun L, Wang X, Zhu Y, Wang Z, Huang Z et al: Double heterozygous mutations of MITF and PAX3 result in Waardenburg syndrome with increased penetrance in pigmentary defects. Clin Genet 83: 78-82, 2013.

2. Read AP and Newton VE: Waardenburg syndrome. J Med Genet 34: 656-665, 1997.

3. Wang J, Li S, Xiao X, Wang P, Guo X and Zhang Q: PAX3 mutations and clinical characteristics in Chinese patients with Waardenburg syndrome type 1. Mol Vis 16: 1146-1153, 2010.

4. Wildhardt G, Zirn B, Graul-Neumann LM, Wechtenbruch J, Suckfüll M, Buske A, Bohring A, Kubisch C, Vogt S, Strobl-Wildemann G, et al: Spectrum of novel mutations found in Waardenburg syndrome types 1 and 2: Implications for molecular genetic diagnostics. BMJ Open 3: e001917, 2013.

5. Kapoor S, Bindu PS, Taly AB, Sinha S, Gayathri N, Rani SV, Chandak GR and Kumar A: Genetic analysis of an Indian family with members affected with Waardenburg syndrome and Duchenne muscular dystrophy. Mol Vis 18: 2022-2032, 2012.

6. Yang SZ, Dai P, Liu X, Kang DY, Zhang X, Yang WY, Zhou CY Yang SM and Yuan HJ: Genetic and phenotypic heterogeneity in Chinese patients with Waardenburg syndrome types II. PLoS One 8: e77149, 2013.

7. Pingault V, Ente D, Dastot-Le Moal F, Goossens M, Marlin S and Bondurand N: Review and update of mutations causing Waardenburg syndrome. Hum Mutat 31: 391-406, 2010.

8. Chaoui A, Watanabe Y, Touraine R, Baral V, Goossens M, Pingault $\mathrm{V}$ and Bondurand $\mathrm{N}$ : Identification and functional analysis of SOX10 missense mutations in different subtypes of Waardenburg syndrome. Hum Mutat 32: 1436-1449, 2011.

9. Iso M, Fukami M, Horikawa R, Azuma N, Kawashiro N and Ogata T: SOX10 mutation in Waardenburg syndrome type II. Am J Med Genet A 146A: 2162-2163, 2008.

10. Zhang H, Luo H, Chen H, Mei L, He C, Jiang L, Li JD and Feng Y: Functional analysis of MITF gene mutations associated with Waardenburg syndrome type 2. FEBS Lett 586: 4126-4131, 2012.

11. Wei XM, Jin F, Ye YH, Xu CM, Qu N, Ju XC and Yi X: A novel mutation of IDS gene in a Chinese patient with mucopolysaccharidosis II by next-generation sequencing. Clinica Chimica Acta 412: 2340-2343, 2011.

12. Wei X, Ju X, Yi X, Zhu Q, Qu N, Liu T, Chen Y, Jiang H, Yang G, Zhen R, et al: Identification of sequence variants in genetic disease-causing genes using targeted next-generation sequencing. PLoS One 6: e29500, 2011.

13. Li H and Durbin R: Fast and accurate long-read alignment with Burrows-Wheeler transform. Bioinformatics 26: 589-595, 2010.

14. Farrer LA, Grundfast KM, Amos J, Arnos KS, Asher JH Jr, Beighton P, Diehl SR, Fex J, Foy C, Friedman TB, et al: Waardenburg syndrome (WS) type I is caused by defects at multiple loci, one of which is near ALPP on chromosome 2: First report of the WS consortium. Am J Hum Genet 50: 902-913, 1992. 